\title{
Effects of Denture Base Thicknesses and Reinforcement on Fracture Strength in Mandibular Implant Overdenture with Bar Attachment: Under Various Acrylic Resin Types
}

\author{
Selen Tokgoz ${ }^{1} \quad$ Arda Ozdiler $^{1} \quad$ Burc Gencel $^{2} \quad$ Ergun Bozdag $^{3} \quad$ Gulbahar Isık-Ozkol ${ }^{1}$
}

${ }^{1}$ Department of Prosthodontics, School of Dentistry, University of Istanbul, Istanbul, Turkey

2Department of Prosthodontics, School of Dental Prosthesis

Technologies, University of Istanbul, Istanbul, Turkey

${ }^{3}$ Department of Mechanical Engineering, Laboratory of

Biomechanics and Strength of Materials, Istanbul Technical

University, Istanbul, Turkey

Eur J Dent 2019;13:64-68
Address for correspondence Selen Tokgoz, DDs, PhD. Department of Prosthodontics, School of Dentistry, Istnabul Okan University, Istanbul 34947, Turkey (e-mail: selenergin@gmail.com).

\begin{abstract}
Keywords

- denture base

thickness

- dynamic loading

- overdenture

Objective This study aimed to assess the effect of reinforcement, denture base thickness, and acrylic resin types on dynamic and static fracture strength in mandibular implant overdentures with bar attachment.

Materials and Methods One hundred and eight experimental mandibular implant overdentures with bar attachments were fabricated in three main groups, namely unreinforced (control: C), reinforced with unidirectional glass fibers (FR), and $\mathrm{Co}-\mathrm{Cr}$ cast metal (MR). Each group included denture bases of 2-, 3-, and 4-mm thicknesses and produced with conventional (CA) and high-impact acrylic (HIA) resins. Specimens were thermocycled $\left(5,000\right.$ times, $\left.5-55^{\circ} \mathrm{C}\right)$ then subjected to a 400,000 cyclic load regime. Unbroken specimens were then loaded until fracture by a universal testing machine. Differences in mean fracture resistance among the groups were compared using the one-way analysis of variance (with post hoc Tukey's honestly significant difference test) and Student's t-tests $(\alpha=0.05)$.

Results Fracture strength increased significantly when the denture base thickness was increased $(p=0.001)$. The 2 -mm denture base thickness was not enough for reinforcement. The fracture strength of the FR groups was significantly higher than other groups for 3 - and $4-\mathrm{mm}$ thicknesses $(p=0.001)$. The fracture strength of the HIA resin was significantly higher than CA resin in FR groups ( $p=0.029$ and $p=0.001)$. MR groups showed the weakest fracture strength.

Conclusions The 2-mm denture base thickness had sufficient fracture strength without reinforcement and a positive relationship between acrylic resin thickness and fracture resistance was found.
\end{abstract}

\section{Introduction}

The treatment of edentulous patients with implant-supported overdenture is a common practice in dentistry; however, denture base fracture is frequent. Fractures usually occur after repeated flexing of the denture base under small loads. Repetitive forces result in the development of microscopic cracks in the areas of biomechanical stress concentration, and it is identified as the main reason for denture base fracture. ${ }^{1,2}$ It is also reported that the thinnest acrylic resin areas which 
are around the copings of overdentures are responsible for fracture. $^{3-10}$ Since biomechanical stresses are focused on these areas, it is suggested that where the reinforcement is necessary. $^{7}$

To understand fracture mechanism of overdentures, dynamic loading tests are important and the number of these tests is limited. ${ }^{11,12}$ Furthermore, there is no published literature about the effect of acrylic resin thickness around the bar attachment. This study aimed to assess the effect of reinforcement, denture base thickness, and acrylic resin types on dynamic and static fracture strength in mandibular implant overdentures with bar attachments. The null hypothesis was that reinforcement materials, denture base thickness, and acrylic resin types would have no effect on the dynamic and static fracture strength of mandibular implant overdenture with bar attachment.

\section{Materials and Methods}

A total of 108 experimental mandibular implant overdentures (EMOs) were fabricated into three main groups, namely unreinforced (control: $\mathrm{C}$ ), reinforced with unidirectional glass fibers (FR), and $\mathrm{Co}-\mathrm{Cr}$ cast metal (MR). Each of the main groups included 2-, 3-, and 4-mm denture base thicknesses and fabricated with conventional (CA) and high-impact (HIA) acrylic resin. A power analysis of pilot data indicated a minimum specimen size of 6 per group $(\alpha=0.05, p=0.80)$.

An edentulous mandibular model was constructed in epoxy resin (Epofix Kit, Struers ApS, Ballerup, Denmark). Two bone-level implants with $60^{\circ}$ conical-internal octagonal connections (Trias, Servo-Dental GMBH, Hagen, Germany) were placed $(4.1 \times 12 \mathrm{~mm})$ at the positions of the canines. To simulate the viscoelasticity of the mucosa, the surface of the residual ridge and the retromolar pad were ground out to a depth of 2 and $4 \mathrm{~mm}$. An artificial mucosa was fabricated (Fit Checker, GC Corporation, Tokyo, Japan) and adapted to epoxy resin model. A Hader-type bar attachment (Bredent, $\mathrm{GmbH}$ \& Co. KG, Senden, Germany) screwed onto the implants. The epoxy resin model was duplicated for producing dental stone models.

A placeholder was fabricated at a thickness of $1.5 \mathrm{~mm}$ to provide sufficient space for the reinforcing material $(0.5 \mathrm{~mm})$ and the acrylic resin $(1 \mathrm{~mm})$. It was length to mesial sides of the first molars over the bar attachment surface (-Fig. 1).

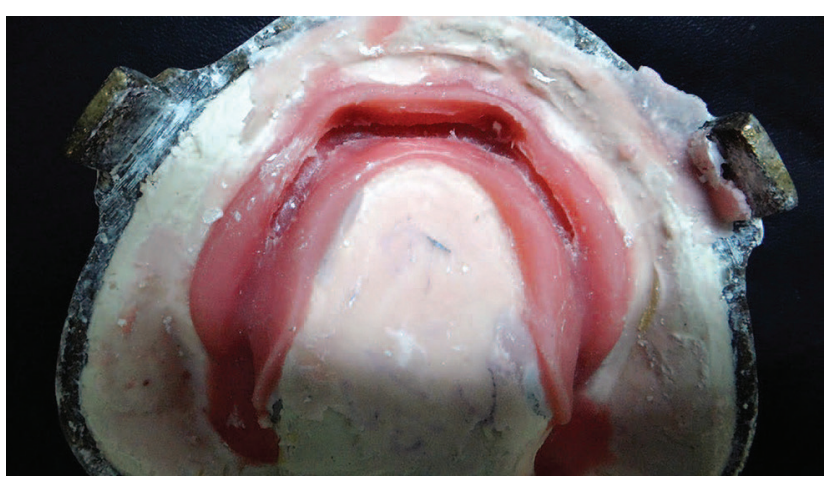

Fig. 1 The space after the placeholder was removed.
Unidirectional glass FR (Stick Tech Ltd, Turku, Finland) and Co-Cr cast MR (Magnum H60, Mesa, Travagliato, Italy) were used as a reinforcing material $(0.5 \mathrm{~mm}$ in thickness and $4 \mathrm{~mm}$ in width). Co-Cr cast MR were sandblasted with $110-\mu \mathrm{m}$ grain-sized alumina for 10 seconds at $0.3 \mathrm{MPa}$ emission pressure, and a MR conditioner (Alloy Primer, Kuraray Medical Inc., Tokyo, Japan) was applied.

For producing identical denture bases, thermoplastic sheets were used at 2-, 3-, and 4-mm thicknesses (Orthotechnology, Thermal Forming Splint, Florida, United States). The denture bases were adapted onto the dental stone model and they were invested in flasks (-Fig. 1). Their surface was covered with a silicon impression material (Elite HD, Zhermack, Italy) to be used again. Acrylic resins were mixed according to the manufacturer's instructions. Polymerization was performed at $70^{\circ} \mathrm{C}$ water bath for 7 hours and at $100^{\circ} \mathrm{C}$ water bath for 1 hour (-Fig. 2).

Specimens were stored in distilled water for 7 days before being thermocycled at 5 to $55^{\circ} \mathrm{C}$ for 5,000 cycles (30 seconds dwell time). Specimens were then subjected to a 400,000 load regime which is equivalent to using a denture for 20 months. ${ }^{13}$ The average fatigue load has been cited as 43 to $100 \mathrm{~N} .{ }^{14}$ In line with this, vertical loading of $98 \mathrm{~N}$ was used in this study and hence the loading delivered to the first molar on the one side was approximately $49 \mathrm{~N}$. Dynamic loading was performed (CS-4.8; SD Mechatronik, Feldkirchen-Westerham, Germany) in a chewing simulator (-Fig. 3) with $1.2 \mathrm{~Hz}$ in distilled water.

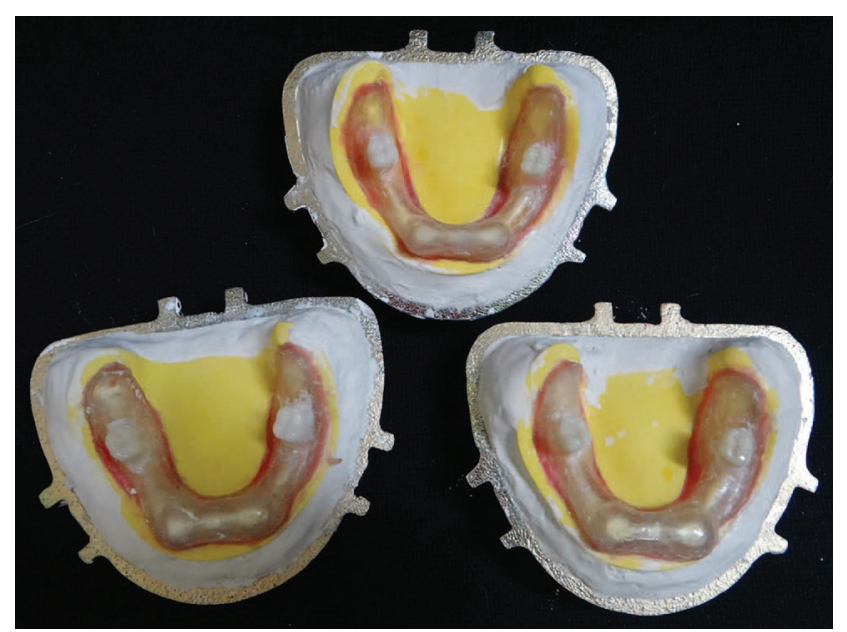

Fig. 2 Thermoplastic denture bases at different thickness.

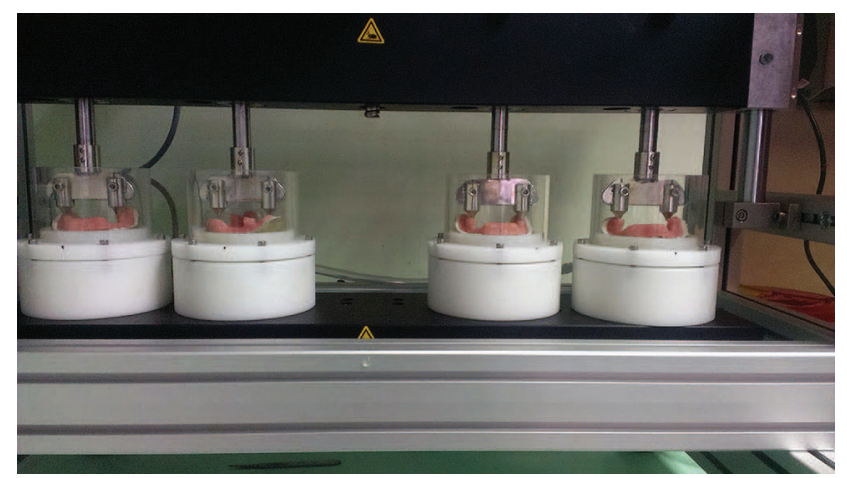

Fig. 3 Test specimens in chewing simulator. 
A concentric cyclic loading was performed. Dynamic loading performed vertically $2 \mathrm{~mm}$ away from the central fossa with 2-mm horizontal sliding motion for imitating chewing procedure. Unbroken specimens were then loaded until fracture by a universal testing machine (MTS, 858 mini Bionix II, Eden Prairie, Minneapolis, United States) at a crosshead speed of $2 \mathrm{~mm} / \mathrm{min}$ (-Fig. 4). During the natural chewing function, especially molar clench, mandible shows horizontal and vertical bending and this function causes torsion of the mandible horizontally. ${ }^{15}$ To mimic horizontal and vertical bending of the mandible, a stainless steel trapezoid tip with a $45^{\circ}$ base angle was designed. The static load applied through this trapezoid tip. This trapezoid tip applied a simultaneous and equal static load to the lingual surfaces of the first molar teeth at an angle of $45^{\circ}$ to imitate static loads occurring in a natural molar clench ( - Fig. $\mathbf{5}$ ).

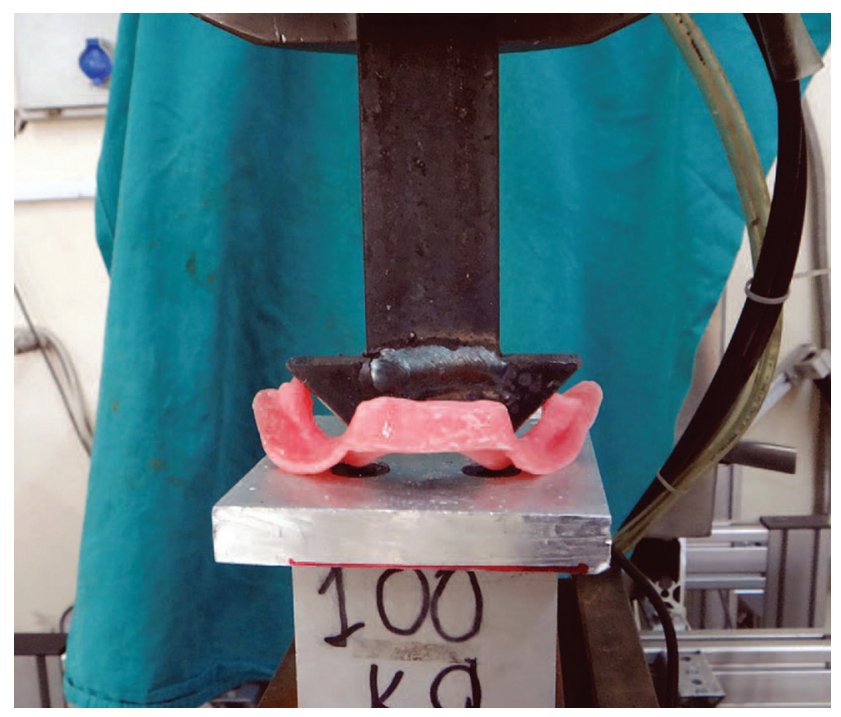

Fig. 4 Fracture apparatus which mimics the mandibular bending in horizontally and vertically.

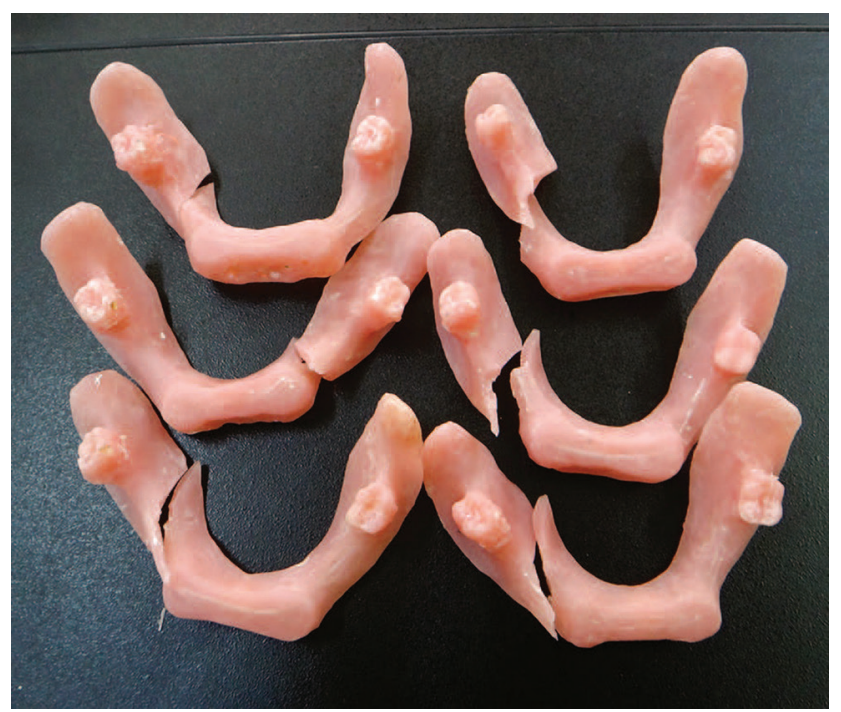

Fig. 5 Sample of fractured fiber specimens.
All tests were performed using statistical software (IBM SPSS, Version 22.0, Armonk, New York, United States). The distribution of the test results was evaluated using the Shapiro-Wilk test. Differences in mean fracture resistance among the groups were compared using the one-way analysis of variance (with post hoc Tukey's honestly significant difference test) and Student's $t$-tests $(\alpha=0.05)$.

\section{Results}

All specimens survived from the dynamic loading. C groups showed the highest fracture strength against the reinforced groups in $2-\mathrm{mm}(\mathrm{CA}=355.99 \pm 28.16$ and $\mathrm{HIA}=469.78 \pm$ 30.4) thicknesses. The 2-mm denture base thickness was found insufficient for any of the reinforcing methods. Fracture strength increased significantly when the denture base thickness was increased $(p=0.001)$. The fracture strength of HIA resin was significantly higher than CA resin in FR groups for all thicknesses $(p=0.001, p=0.029$, and $p=0.001)$. No significant difference was found in HIA and CA resins in $C$ $(p=0.879$ and $p=0.564)$ and MR groups $(p=0.627$ and $p=$ 0.804 ) at 3 - and $4-\mathrm{mm}$ thicknesses. MR groups appeared to be the weakest groups ( - Table $\mathbf{1}$ ).

\section{Discussion}

It was hypothesized that the reinforcement materials, denture base thickness, and acrylic resin types would have no effect on the dynamic and static fracture strength of mandibular implant overdenture with bar attachment. The statistical analysis showed that the null hypothesis for dynamic strength should not be rejected but for static strength should be rejected.

The 2-mm thick specimens were found too thin to pack the amount of acrylic resin necessary for efficient impregnation of the reinforcements. C groups showed significantly higher fracture strength than reinforced groups at 2-mm denture base thickness. This finding in combination with the available literature implies that care should be given to keep the denture base thickness at a minimum of $2 \mathrm{~mm}$ even if there is limited space for the resin. In case of limited interalveolar distance, the artificial tooth can be adjusted to provide a 2-mm space for the acrylic resin to avoid any fracture failures. Furthermore, the design of both the bar attachment and the denture base can be made with one of the indexing techniques to keep the resin thickness at $2 \mathrm{~mm}$ around the attachment. According to the current study result, denture base thickness significantly affects the fracture strength of the EMOs. The positive relationship between acrylic resin thickness and fracture strength was confirmed in accordance with the previous studies. $3,6,16$

MR groups showed the lowest fracture strength in all groups. The result of the current study is similar to those reported by others. ${ }^{17,18}$ This finding may be attributed to the foreign body effects of MR reinforcement on acrylic resin. ${ }^{12}$ Cast MR inserts, even if they are physically and/or chemically treated, do not provide reinforcement to the denture base; instead, they weaken the overall structure by reducing the 
Table 1 Comparisons of fracture strength means and SD among ( $\mathrm{n})$ in specimen groups

\begin{tabular}{|l|l|l|l|l|}
\hline \multirow{2}{*}{$\begin{array}{l}\text { Reinforced and } \\
\text { unreinforced Groups }\end{array}$} & Thickness $(\mathrm{mm})$ & \multicolumn{2}{|c|}{ Mean \pm SD } & \multirow{2}{*}{$p$-Value } \\
\cline { 3 - 5 } & & CA & HIA & \\
\hline C & 2 & $355.99 \pm 28.16$ & $469.78 \pm 30.4$ & $0.001^{\text {a }}$ \\
\hline MR & 2 & $211.66 \pm 44.49$ & $404.64 \pm 57.05$ & $0.001^{\text {a }}$ \\
\hline FR & 2 & $311.6 \pm 41.15$ & $519.54 \pm 41.64$ & $0.001^{\text {a }}$ \\
\hline C & & $0.001^{\mathrm{b}}$ & 0.001 & \\
\hline MR & 3 & $467.09 \pm 29.26$ & $534.50 \pm 62.06$ & 0.879 \\
\hline FR & 3 & $452.4 \pm 21.8$ & $457.56 \pm 12.63$ & 0.627 \\
\hline$P$ & 3 & $620.86 \pm 67.85$ & $704.6 \pm 13.5$ & $0.029^{\text {a }}$ \\
\hline C & & $0.001 \dagger$ & $0.001^{\mathrm{b}}$ & \\
\hline MR & 4 & $803.48 \pm 40.34$ & $780.35 \pm 85.92$ & 0.564 \\
\hline FR & 4 & $762.19 \pm 14.63$ & $765.53 \pm 28.47$ & 0.804 \\
\hline$P$ & 4 & $865.54 \pm 14.5$ & $917.7 \pm 20.65$ & $0.001^{\text {a }}$ \\
\hline
\end{tabular}

Abbreviations: ANOVA, analysis of variance; C, control; CA, conventional; FR, fibers; HIA, high impact; MR, metal; SD, standard deviation.

aStudent's t-test.

'One-way ANOVA test $p<0.05$.

thickness of the resin. FR groups showed the highest fracture strength at 3- and 4-mm thick EMOs. These findings were similarly with previous studies. ${ }^{19-24}$

According to previous studies, the performance of HIA resins has not been investigated comparatively below $2.5-\mathrm{mm}$ thickness, where it is actually critical. The specimen geometry and loading protocols up-to-date were far from being able to generate similar strains as an overdenture under function..$^{21,25-28}$ Thus, test specimens and loading protocol were designed in a different methodology. In the current study, HIA resin showed significantly higher fracture strength than CA resin in 2-mm thickness. Furthermore, HIA resin showed significantly higher fracture strength than CA resin in 3- and 4-mm thicknesses for FR groups. Hamouda and Beyari reported in a study that HIA resins with FR reinforcement showed better flexural strength than CA resin. ${ }^{21}$ FR reinforcement may affect the fracture strength of HIA resin, but further studies are needed. The fracture strength was not significantly different between HIA and CA resin for MR and C groups at 3- and 4-mm thicknesses, respectively. These results were similar with previous studies. 25,26,28

There are several limitations in this study. There is no standard protocol or information exists about when a dynamic loading should be performed after the thermocycling in previous studies or when a static test should be performed after the dynamic loading test. ${ }^{11,12}$ For the current study, dynamic loading test was performed within 24 hours, after the thermocycling and static test was performed within 48 hours after the dynamic loading test performed. All specimens were stored in distilled water at room temperature until the dynamic loading began and over. The oral conditions cannot be accurately simulated, although the mandibular models were fabricated in epoxy resin, which has the similar young modulus with the mandibular jaw. EMOs were supported by epoxy models during the dynamic test, which may not show the same flexibility as that of the mandible in function. Although the dynamic test was performed in wet conditions, the Ph of the oral environment could not mimic exactly. This is the first study of this kind; hence, further in vitro studies needed to validate the result of the present study. However, the findings as well as the procedures used in this study may aid researchers in conducting more studies that mimic the clinical conditions more closely in the future.

\section{Conclusions}

Within the limitations of this study, 2-mm denture base thickness had sufficient fracture strength without reinforcement and a positive relationship between acrylic resin thickness and fracture resistance was found.

\section{Financial Support and Sponsorship}

This work was supported by Scientific Research Projects Coordination Unit of Istanbul University (Project No: 2097-47444 and ONAP 1509-42829). Finally, the author, Selen Tokgöz, thanks TUBITAK-2211 for supporting the $\mathrm{PhD}$ education.

\section{Conflicts of Interest}

None declared.

\section{References}

1 Jagger DC, Harrison A, Jandt KD. The reinforcement of dentures. J Oral Rehabil 1999;26(3):185-194

2 Khuder T, Yunus N, Sulaiman E, Dabbagh A. Finite element analysis and clinical complications in mandibular implantoverdentures opposing maxillary dentures. J Mech Behav Biomed Mater 2017;75:97-104

3 Choi M, Acharya V, Berg RW, et al. Resinous denture base fracture resistance: effects of thickness and teeth. Int J Prosthodont 2012;25(1):53-59 
4 Duncan JP, Freilich MA, Latvis CJ. Fiber-reinforced composite framework for implant-supported overdentures. J Prosthet Dent 2000;84(2):200-204

5 Rodrigues AH. Metal reinforcement for implant-supported mandibular overdentures. J Prosthet Dent 2000;83(5):511-513

6 Fajardo RS, Pruitt LA, Finzen FC, Marshall GW, Singh S, Curtis DA. The effect of E-glass fibers and acrylic resin thickness on fracture load in a simulated implant-supported overdenture prosthesis. J Prosthet Dent 2011;106(6):373-377

7 Dong J, Ikebe K, Gonda T, Nokubi T. Influence of abutment height on strain in a mandibular overdenture. J Oral Rehabil 2006;33(8):594-599

8 Takahashi T, Gonda T, Maeda Y. Influence of reinforcement on strains within maxillary implant overdentures. Int J Oral Maxillofac Implants 2015;30(6):1327-1332

9 Gonda T, Ikebe K, Dong J, Nokubi T. Effect of reinforcement on overdenture strain. J Dent Res 2007;86(7):667-671

10 Agha $H$, Flinton R, Vaidyanathan T. Optimization of fracture resistance and stiffness of heat-polymerized high impact acrylic resin with localized E-glass FIBER FORCE® reinforcement at different stress points. J Prosthodont 2016;25(8):647-655

11 Im SM, Huh YH, Cho LR, Park CJ. Comparison of the fracture resistances of glass fiber mesh- and metal mesh-reinforced maxillary complete denture under dynamic fatigue loading. J Adv Prosthodont 2017;9(1):22-30

12 Rached RN, de Souza EM, Dyer SR, Ferracane JL. Dynamic and static strength of an implant-supported overdenture model reinforced with metal and nonmetal strengtheners. J Prosthet Dent 2011;106(5):297-304

13 Steiner M, Mitsias ME, Ludwig K, Kern M. In vitro evaluation of a mechanical testing chewing simulator. Dent Mater 2009;25(4):494-499

14 Fontijn-Tekamp FA, Slagter AP, Van Der Bilt A, et al. Biting and chewing in overdentures, full dentures, and natural dentitions. J Dent Res 2000;79(7):1519-1524

15 Favot LM, Berry-Kromer V, Haboussi M, Thiebaud F, Ben Zineb T. Numerical study of the influence of material parameters on the mechanical behaviour of a rehabilitated edentulous mandible. J Dent 2014;42(3):287-297

16 Sekinishi T, Inukai S, Murakami N, Wakabayashi N. Influence of denture tooth thickness on fracture mode of thin acrylic resin bases: an experimental and finite element analysis. J Prosthet Dent 2015;114(1):122-129
17 Heidari B, Firouz F, Izadi A, Ahmadvand S, Radan P. Flexural strength of cold and heat cure acrylic resins reinforced with different materials. J Dent (Tehran) 2015;12(5):316-323

18 Yoshida K, Takahashi Y, Shimizu H. Effect of embedded metal reinforcements and their location on the fracture resistance of acrylic resin complete dentures. J Prosthodont 2011;20(5):366-371

19 Yoshida K, Takahashi Y, Hamanaka I, Kawaguchi T, Sasaki H, Shimizu H. Reinforcing effect of glass fiber-reinforced composite reinforcement on flexural strength at proportional limit of a repaired denture base resin. Acta Biomater Odontol Scand 2015;1(2-4):81-85

20 Vallittu PK. Curing of a silane coupling agent and its effect on the transverse strength of autopolymerizing polymethylmethacrylate-glass fibre composite. J Oral Rehabil 1997;24(2):124-130

21 Hamouda IM, Beyari MM. Addition of glass fibers and titanium dioxide nanoparticles to the acrylic resin denture base material: comparative study with the conventional and high impact types. Oral Health Dent Manag 2014;13(1):107-112

22 Alla R, Sajjan S, Alluri V, Ginjupalli K, Padhya NU. Influence of fiber reinforcement on the properties of denture base resins. J Biomed Nanotechnol 2013;4:91-97

23 Singh K, Sharma SK, Negi P, Kumar M, Rajpurohit D, Khobre P. Comparative evaluation of flexural strength of heat polymerized denture base resins after reinforcement with glass fibers and nylon fibers: an in vitro study. Adv Hum Biol 2016;6:91-94

$24 \mathrm{Yu} \mathrm{SH}$, Cho HW, Oh S, Bae JM. Effects of glass fiber mesh with different fiber content and structures on the compressive properties of complete dentures. J Prosthet Dent 2015;113(6):636-644

25 Jagger DC, Jagger RG, Allen SM, Harrison A. An investigation into the transverse and impact strength of "high strength" denture base acrylic resins. J Oral Rehabil 2002;29(3):263-267

26 Ajaj-Alkordy NM, Alsaadi MH. Elastic modulus and flexural strength comparisons of high-impact and traditional denture base acrylic resins. Saudi Dent J 2014;26(1):15-18

27 Johnston EP, Nicholls JI, Smith DE. Flexure fatigue of 10 commonly used denture base resins. J Prosthet Dent $1981 ; 46(5): 478-483$

28 Gupta A, Tewari RK. Evaluation and comparison of transverse and impact strength of different high strength denture base resins. Indian J Dent Res 2016;27(1):61-65 